\title{
Classification Based on LBP and SVM for Human Embryo Microscope Images
}

\author{
Yabo Yin ${ }^{1}$, Yun Tian ${ }^{1}$, Weizhong Wang ${ }^{2}$, Fuqing Duan ${ }^{1}$, \\ Zhongke $\mathrm{Wu}^{1}$, and Mingquan $\mathrm{Zhou}^{1}$ \\ ${ }^{1}$ College of Information Science and Technology, Beijing Normal University, Beijing, China \\ ${ }^{2}$ Reproductive Medicine Center, Navy General Hospital, PLA, Beijing, China \\ yinyabo0612@163.com
}

\begin{abstract}
Embryo transfer is an extremely important step in the process of invitro fertilization and embryo transfer (IVF-ET). The identification of the embryo with the greatest potential for producing a child is a very big challenge faced by embryologists. Most current scoring systems of assessing embryo viability are based on doctors' subjective visual analysis of the embryos' morphological features. So it provides only a very rough guide to potential. A classifier as a computer-aided method which is based on Pattern Recognition can help to automatically and accurately select embryos. This paper presents a classifier based on the support vector machine (SVM) algorithm. Key characteristics are formulated by using the local binary pattern (LBP) algorithm, which can eliminate the inter-observer variation, thus adding objectivity to the selection process. The experiment is done with 185 embryo images, including 47 "good" and 138 "bad" embryo images. The result shows our proposed method is robust and accurate, and the accurate rate of classification can reach about $80.42 \%$.
\end{abstract}

Keywords: embryo microscope images, feature extraction, automatic classifier, local vector pattern, support vector machine.

\section{Introduction}

Nowadays, the efficiency of single embryo transfer remains relatively poor. In order to improve the odds of a successful pregnancy, it is common to transfer more than one to the uterus per cycle, but this often results in multiple pregnancies (twins, triplets, etc), which are associated with significantly elevated risks of serious complications [1]. How to recognize viable embryos remains a big challenge. Most current methods of embryo viability assessment are based on doctors' subjective visual analysis of the embryos' morphological features [2], and tend to be subjective and imprecise. Moreover, the ability of human's eye is so limited that some important but unintuitive information cannot be identified. Aiming at this problem, some researchers [3, 4] attempted to identify viable embryos with the help of the pattern recognition methods, and they identified embryo images into two classes: those suitable for procreation and those not suitable, by designing a decision support system. So the problem will be 
simplified into two sub-problems. One is to get the feature vectors that are significantly different between two classes. The other is to design a good classifier with a high accuracy rate. Patrizi et al.[5] presented the TRACE algorithm, which recognized embryos as belonging to one of the two classes. The pattern vector of embryo features is comprised of 10 moments calculated based on the embryo image histogram. The classification algorithm used training sets to establish centers of classes and classification was then performed based upon a measure of distance from the class centers. In tests on 165 images, the average accuracy was claimed up to 0.85. Morales et al. [6] developed a decision support system based on a Bayesian classifier. The feature vector that describes each embryo image consists of variables based on morphology and on the clinical data of the patients. In tests on 63 cases, using different types of Bayesian classifiers, the accuracy of the systems was claimed from $63.49 \%$ to $71.43 \%$. These classifiers greatly depend on the experiment datasets and may be improper to other data, although good for theirs.

The feature extraction of embryo images has a very big influence on the classification, so finding a valid description of the images is a key step for classifying the embryos. Some commonly used feature extraction methods in pattern recognition, such as principal component analysis (PCA) [7] or linear discriminant analysis (LDA) [8], mostly depict an image from the overall point of view, which can well extract the global properties of the image but are sensitive to light and position. Moreover, the feature extraction method based on central moments $[5,9]$ which has been used by some previous researches can quantify the key characteristics of embryo images in some extent, but it fails to consider the difference in the light intensity of embryo images. The LBP algorithm is not sensitive to light and can well extract the local texture of an image. It was first put forward by Ojala et al. [10], and it is widely used in texture analysis, such as face recognition. However, in this paper, we first employ the LBP algorithm to analyze human embryo images.

Designing a well-performed classifier is the other key step to ensure the recognition rate. Since SVM shows a superior performance in learning from a small number of samples, the classifier designed in this paper is based on this algorithm. In addition, we also use another classical pattern recognition method-K-NN [11] to design a classifier as a contrast. These classifiers will make use of our first-hand embryo images, which are all from fresh transfer cycles. Most of the images (about 90\%) are first used to train the classifier, and then the rest to make a test on the basis of the rule learnt in training.

\section{Methodology}

\subsection{Feature Extraction Based on LBP}

The LBP algorithm is defined as a gray-scale invariant texture measure, derived from a general definition of texture in a local neighborhood. This algorithm is a non-parametric kernel which summarizes the local spatial structure of an image. 
Moreover, it is invariant to monotonic gray-scale transformations. Hence the LBP representation may be less sensitive to changes in illumination. This is a very interesting property in embryo microscope image recognition. Almost all the state-of-the-art embryo image recognition algorithms are based on statistical classifier and local image features, which are noise sensitive and hardly to deliver perfect recognition performance. We have developed a novel feature extraction method, using the histogram of local binary pattern for global embryo image texture representation.

Fig. 1 describes the basic LBP algorithm. Fig. 1 (a) is a $3 \times 3$ rectangular area. First take its center pixel grayscale value as the closed value, and then compare its neighborhood eight pixels. If the center pixel value is less than its adjacent pixel value, we will set the value of this adjacent pixel as 1 , otherwise set it as 0 , so to produce the binary code of the region. As shown in Fig.1 (b), this model can be described by eight binary codes. Convert the binary code to a decimal number, and we obtain the LBP code of the center pixel, as shown in Fig.1 (c). The corresponding LBP code of each pixel reflects its neighborhood gray distribution. The LBP code histogram of certain region of an image can be used to describe the regional texture structure.

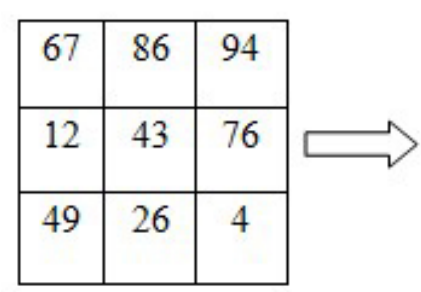

(a)

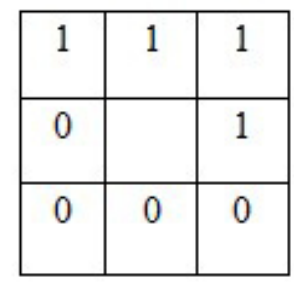

(b)

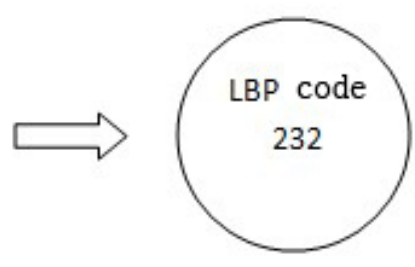

(c)

Fig. 1. The transformation process of LBP. (a) $3 \times 3$ pixels with different grayscale value ;(b) eight binary codes; (c) LBP code of the center pixel.

Fig. 2 shows the results of applying LBP algorithm to extract features of different images. After calculating every pixel's corresponding LBP code, we do a frequency count for all the LBP code to produce a new gray histogram, and finally extract a feature vector by $1 * 256$. 


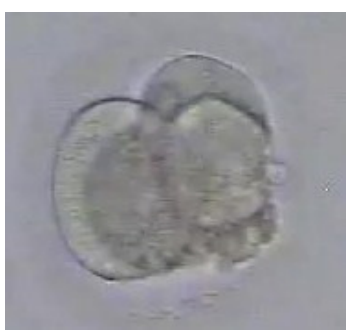

(a)

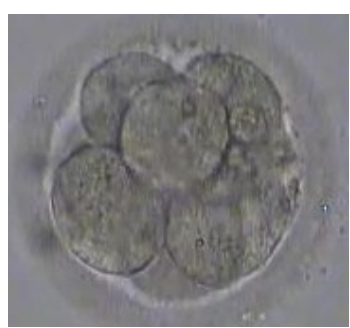

(c)

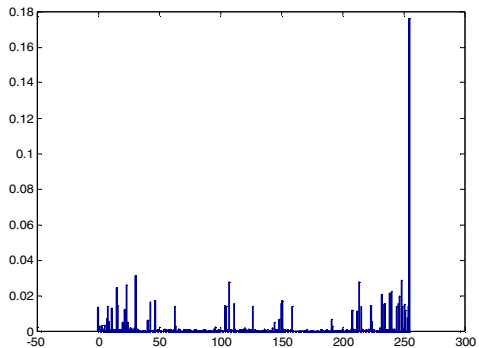

(b)

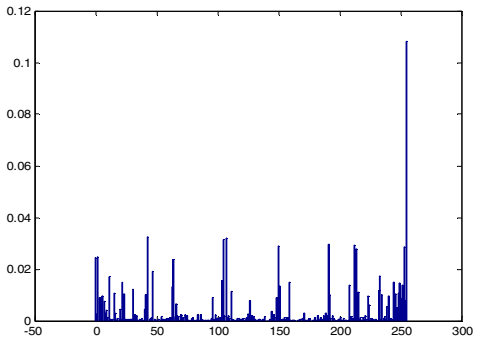

(d)

Fig. 2. LBP histograms of different images. (a) a "bad" embryo image;(b) LBP histogram corresponding to (a); (c) a "good" embryo image;(d) LBP histogram corresponding to (c).

\subsection{Classifier Design}

The SVM algorithm $[12,13]$ is based on the statistical learning theory and the principle of minimum structural risk. It seeks an optimal compromise between the complexity of the model (i.e. the accuracy of studying pending training samples) and the learning ability (i.e. no wrong recognition ability of arbitrary sample) according to the limited sample information, so as to get the best promotion ability. The SVM aims at finite samples and the sample size is small in this paper, so it is proper to be employed to classify the microscope embryo images.

Considering a model classifier, the training set is given, $x_{i}$ is a vector of the input space, and $y_{i}$ is the classification identification, $y_{i} \in\{-1,1\}$. If the input vector set is linearly separable, then they can be set apart by a hyperplane $w^{*} x_{i}-b=0$ ( $w$ is the normal vector of the classification hyperplane; $b$ denotes the offset).If this vector set was correctly set apart by the hyperplane and the distance between the nearest vector from the hyperplane and the hyperplane is the longest, then the vector set is divided by the optimal hyperplane. In order to describe the classification hyperplane, we use the following form: 


$$
\left\{\begin{array}{l}
w * x_{i}-b \geq 1 \quad \text { if } \quad y_{i}=1 \\
w * x_{i}-b \leq-1 \quad \text { if } \quad y_{i}=-1
\end{array}\right.
$$

According to the minimum structural risk (SRM) principle, looking for the biggest interval hyperplane is just looking for the smallest VC dimension to minimize the confidence interval. So by solving the following optimization problem, SVM obtains the optimal classification hyperplane:

$$
\min \frac{1}{2}\|w\|^{2} \quad \text { s.t } y_{i}[w * x-b] \geq 1(i=1,2, \ldots, l)
$$

In order to construct the optimal classification hyperplane when the data is linearly inseparable, we introduce the nonnegative variables $\xi_{i}>0$ to construct a weak and indirect optimal hyperplane:

$$
\min \frac{1}{2}\|w\|^{2}+C\left(\sum_{i=1}^{l} \xi_{i}\right) \quad \text { s.t } y_{i}[w * x-b] \geq 1-\xi_{i}\left(i=1,2, \ldots, l, \xi_{i} \geq 0\right)
$$

where $\mathrm{C}$ is a regular parameter.

For the linearly inseparable problem, the SVM method maps the input space to a high-dimension feature space through nonlinear mapping, and then constructs the optimal hyperplane in the high-dimension feature space. SVM converts the inner product operation into the calculation in the input space by introducing the kernel function, namely $K\left(x_{i}, x_{j}\right)=\left[\varphi\left(x_{i}\right), \varphi\left(x_{j}\right)\right]$, where $K\left(x_{i}, x_{j}\right)$ is the kernel function.

So solving SVM is solving the optimization of the following problem:

$$
\begin{gathered}
\max W(a)=\sum_{i=1}^{l} a_{i}-\frac{1}{2} \sum_{i, j=1}^{l} a_{i} \mid a_{j} y_{i} y_{j} K\left(x_{i}, x_{j}\right) ;\left(0 \leq a_{i} ; i=1, \ldots l\right) \\
\text { s.t } \quad \sum_{i=1}^{l} a_{i} y_{i}=0
\end{gathered}
$$

The corresponding decision-making function for classification is:

$$
f(x)=\operatorname{sgn}\left(\sum_{i=s v} y_{i} a_{i}{ }^{0} K\left(x_{i}, x\right)-b_{0}\right)
$$

Here, we use gaussian kernel to be the kernel function. We randomly select about $90 \%$ of the embryo images in each class to train the classifier, and use cross validation to get the optimal classifier parameters. Finally, the classifier is tested with the rest images.

\section{Experiments and Analysis}

This paper uses 385 fresh embryo images, 185 transfer cycles, from assisted reproductive medical center at navy general hospital, as original datasets. They were 
photographed 3 days after fertilization. In each transfer cycle, two or three embryos were transferred into the uterus. Clinical outcome showed that among these $185 \mathrm{emb}-$ ryo transfer cycles, 138 failed, which means, no embryo gave birth in the end. And the other 47 cycles succeeded, that is to say, at least one embryo became a baby in the end.

Considering that the labels of those images from the good class (at least one embryo gave birth) are imprecise, for it is difficult to know which one on earth became a baby in the end. In order to simplify the problem, this paper assumes that all images used in the experiment belong to a certain class for sure. Thus, there are some limitations when picking the images. In this paper, we randomly select one embryo from each transfer cycle. Consequently, we obtain 185 images, 138 of which are from the bad class, and the other 47 are from the good class.

According to our direct visual observation, there is no significant difference in morphological features between the two kinds of images. One possibility is that all these embryos have already been picked out according to their morphological features. Thus it is important to have the aid of pattern recognition methods to further select the viable embryos. Figure 3 shows some of the typical and atypical images from the two classes.

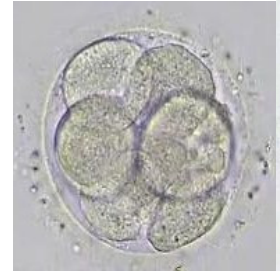

(a)

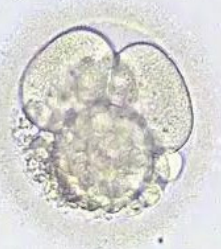

(b)

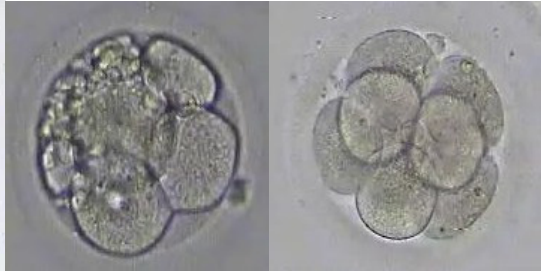

(c) (d)

Fig. 3. Some of the "good" and "bad" images. (a), (b), (c) and (d),typical "good", typical "bad", atypical "good" and atypical "bad" embryo image, respectively.

Table 1 shows the classification results of the K-NN and SVM methods with different feature vectors. We do three groups of experiments for each classification method. They are named as group1, group 2 and group 3. In each group, the classification algorithm is carried out for 10 times with randomly chosen training and testing samples. And we estimate the performance of the classifier according to the classification accuracy of the test. Finally, we calculate the average accuracy of the three groups for each method. 
Table 1. Classification accuracy of different classification methods

\begin{tabular}{|c|c|c|c|c|}
\hline Feature extraction-Classification & Group No. & Mean & Max & Min \\
\hline \multirow{4}{*}{ Central moments-K-NN } & 1 & 0.5278 & 0.7222 & 0.2778 \\
\hline & 2 & 0.6000 & 0.7778 & 0.4444 \\
\hline & 3 & 0.6833 & 0.8889 & 0.5556 \\
\hline & \multicolumn{4}{|c|}{ The average accuracy of three group: 0.6037} \\
\hline \multirow{4}{*}{$\mathrm{LBP}-\mathrm{K}-\mathrm{NN}$} & 1 & 0.6333 & 0.7778 & 0.4444 \\
\hline & 2 & 0.7000 & 0.8889 & 0.5000 \\
\hline & 3 & 0.6556 & 0.7778 & 0.5000 \\
\hline & \multicolumn{4}{|c|}{ The average accuracy of three group: 0.6630} \\
\hline \multirow{4}{*}{$\mathrm{LBP}-\mathrm{SVM}$} & 1 & 0.7964 & 0.8571 & 0.7143 \\
\hline & 2 & 0.8143 & 0.8571 & 0.7143 \\
\hline & 3 & 0.7964 & 0.8214 & 0.6786 \\
\hline & \multicolumn{4}{|c|}{ The average accuracy of three group:0.8024 } \\
\hline
\end{tabular}

Fig. 4 shows the graph of the average accuracy of different classifiers and the comparison chart of two feature extraction methods with K-NN.

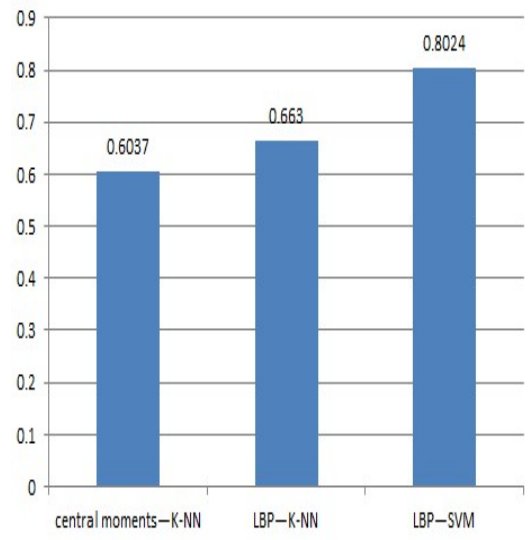

(a)

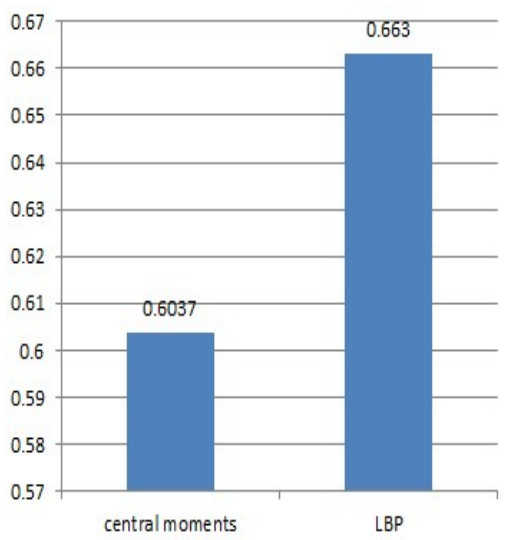

(b)

Fig. 4. Graph of the average accuracy of different classifiers. (a) average classification accuracy of the three classification methods; (b) average classification accuracy of the K-NN classifier with two different feature extraction methods.

From the Fig.4, we can see that LBP algorithm as the feature extraction method is better than that based on central moments. And obviously, SVM algorithm has the 
absolute advantage in classifying our datasets. Not only that the average accuracy of classification by using SVM is as high as 0.8024 , but also that the accuracy is over a small range. In addition, since most classifiers are sensitive to the number of the training samples, especially when the difference between different classes is not significant, too few samples will greatly decrease the performance of the classifier.

However, the performance of these classifiers is not very ideal in the whole compared with other applications. As for the reasons, the first one is that whether an embryo is good or not is not the single factor to decide the possibility of giving a birth. Some other factors, such as the gestational age, the number of pregnant, et al, will also greatly influence the result. Secondly, only one embryo image actually cannot reflect all information of the embryo, thus we cannot make an objective assessment of the embryo's quality just by one image.

\section{Discussion}

Challenge is enormous. First of all, related researches about the classification of the embryo images are relatively rare. Moreover, the few researches greatly depend on the data they use and a classification method with a relatively high accuracy for some data may not be proper for other data. In this paper, a new classification solution is proposed based on the LBP and SVM algorithms. The method has an average accuracy of 0.8024 , and is superior to that based on central moments and K-NN. However, the proposed method still has some aspects to be improved, and if some other morphological features, such as the thickness of zona pellucida or the number of blastomeres et al, are taken into account, the classification result may be more excellent.

In addition, from the aspect of designing the classifier itself, there are some problems to be solved. First, as mentioned above, it is a semi-supervised classification problem, which is difficult to deal with. Secondly, there is no significant morphological difference between the two classes, which makes the feature extraction difficult and negatively influences the performance of the classifier. Thirdly, the number of embryo images available in this experiment is too small and even a little unbalanced between the two classes, which may lead to the classifier not stable.

Acknowledgements. This work is partly supported by the National Natural Science Foundation of China (No.61003134, 61170170) and the Key Program of Beijing Natural Science of Beijing (No. 4081002). The authors would like to thank the anonymous reviewers who gave valuable suggestions that have helped to improve the quality of the manuscript.

\section{References}

1. Santos Filho, E., Noble, J.A., Wells, D.: A Review on Automatic Analysis of Human Embryo Microscope Images. Open Biomed. Eng. J. 4, 170-177 (2010)

2. Santos Filho, E., Noble, J.A., Wells, D.: Toward a Method for Automatic Grading of Microscope Human Embryo Images. In: IEEE International Symposium on Biomedical Imaging, pp. 1289-1292 (2010) 
3. Ning, F., Delhomme, D., LeCun, Y., Piano, F., Bottou, L., Barbano, P.E.: Toward Automatic Phenotyping of Developing Embryos fromVideos. IEEE Trans. Image Process 14(9), 1360-1371 (2005)

4. Siristatidis, C., Pouliakis, A., Chrelias, C., Kassanos, D.: Artificial Intelligence in IVF: A Need. Syst. Biol. Reprod. Med. 57(4), 179-185 (2011)

5. Patrizi, G., Manna, C., Moscatelli, C., Nieddu, L.: Pattern Recognition Methods in Humanassisted Reproduction. Int. Trans. Oper. Res. 11(4), 365-379 (2004)

6. Morales, D.A., Bengoetxea, E., Larranaga, P., et al.: Bayesian Classification for the Selection of in Vitro Human Embryos Using Morphological and Clinical Data. Comput. Meth. Prog. Bio. 90(2), 104-116 (2008)

7. Behdad, M., French, T., Barone, L., Bennamoun, M.: PCA for Improving the Performance of XCSR in Classification of High-dimensional Problems. In: Proceedings of the 13th Annual Conference Companion on Genetic and Evolutionary Computation, pp. 361-368 (2011)

8. Kobayashi, H., Zhao, Q.F.: Face Detection Based on LDA and NN. In: Proceedings of the 2007 Japan-China Joint Workshop on Frontier of Computer Science and Technology, pp. 146-154 (2007)

9. Morales, D.A., Bengoetxea, E., Larrabaga, P.: Selection of Human Embryos for Transfer by Bayesian Classifiers. Comput. Biol. Med. 38(11), 1177-1186 (2008)

10. Ojala, T., Pietikainen, M., Maenpaa, T.: Multiresolution Gray-Scale and Rotation Invariant Texture Classification with Local Binary Patterns. IEEE Trans. PAMI 24(7), 971-987 (2002)

11. Kou, H.Z., Gardarin, G.: Study of Category Score Algorithms for K-NN Classifier. In: Proceedings of the 25th Annual International ACM SIGIR Conference on Research and Development in Information Retrieval, pp. 393-394 (2002)

12. Christopher, J., Burges, C.: A Tutorial on Support Vector Machines for Pattern Recognition. Data Min. Knowl. Disc. 2(2), 121-167 (1998)

13. Wan, H.L., Chowdhury, M.: Image Semantic Classification by Using SVM. JSW 14(11), 1891-1899 (2003) 